\title{
Seismic Fragilities of Curved Concrete Bridges via Bayesian Parameter Estimation Method
}

\author{
Jong-Su Jeon', Taehyo Park ${ }^{2}$ \\ ${ }^{1}$ Andong National University \\ 1375 Gyeongdong-ro, Andong-si, Gyeongsangbuk-do, South Korea \\ jsjeon@anu.ac.kr \\ ${ }^{2}$ Hanyang University \\ 222 Wangsimni-ro, Seongdong-gu, Seoul, South Korea \\ cepark@hanyang.ac.kr
}

\begin{abstract}
In this paper, a Bayesian parameter estimation method is applied for a regional seismic risk assessment of curved concrete bridges. For this purpose, a class of three-frame concrete box-girder bridges is chosen as a case-study bridge. Numerical bridge models accounting for geometric and material uncertainties are simulated in dynamic analyses to construct multi-parameter demand models of bridge components, including various uncertainty parameters and an intensity measure. The demand models are established introducing a Bayesian parameter estimation method. Logistic regression is used to develop parameterized fragility curves comparing demands and capacities. The developed fragilities can be used to generate their bridge-specific fragilities using a specific value of uncertainty parameters. Additionally, bridge-class fragility curves conditioned only on the intensity measure can be developed by integrating the parameterized fragility functions over the domain of the uncertainty parameters to assess the vulnerability of this bridge class. The bridge-class fragility model significantly reduces the model error in comparison to the traditional fragility model.
\end{abstract}

Keywords: curved bridges, multi-parameter demand models, parameterized, bridge-specific and bridge-class fragility curves, Bayesian parameter estimation

\section{Introduction}

Fragility function is the conditional probability of exceeding a specific damage state given a ground motion intensity measure (IM). The seismic fragility of bridges has been traditionally used with bridge-class fragility curves developed with linear or quadratic regression demand models conditioned on a single-parameter (IM) [1-3]. The single-parameter demand model is convolved with a limit state model to calculate a fragility function, a lognormal cumulative distribution function (CDF). However, [4] states that single-parameter demand models and fragility curves have the following limitations: (1) inability to reflect the effect of uncertainty parameters on structural performance during earthquakes without extensive resimulations for each new set of parameter combinations and (2) inability to explicitly address the effect of uncertainty parameters on fragility curves. Recently, to alleviate such limitations of the single-parameter demand models, logistic regression in conjunction with multi-parameter demand models comprising various predictor variables has been developed in the realm of seismic vulnerability and loss estimation [4,5]. [5] employed response surface demand models and logistic regression to derive aging highway bridge fragilities. [4] used various surrogate modeling techniques to decide the bestfitting multi-parameter demand model for bridge fragilities.

As another statistical method, this study applies a Bayesian parameter estimation method to develop multi-parameter demand models. For this purpose, a class of multi-frame concrete bridges is selected, which are representative of curved bridges in California. A set of numerical bridge models are created in OpenSees [6], which includes (1) material and geometric uncertainties based on a bridge inventory analysis and (2) the nonlinear response of various bridge components in the case-study bridges. Using the response data obtained from dynamic analyses and the uncertainty parameters, multiparameter demand models are constructed via the Bayesian parameter estimation method. The demand models and limit state models are employed to perform logistic regression to develop parameterized fragility curves, which will be used to produce bridge-specific fragility curves for the regional risk assessment if uncertainty parameters are available. 
Additionally, bridge-class fragility curves are developed by integrating the parameterized fragility functions over the domain of input parameters if input parameters are probabilistic in nature and used for the comparison with the traditional fragility models.

\section{Fragility Function via Bayesian Parameter Estimation}

\subsection{Bayesian Parameter Estimation}

The unknown unobserved parameter $(\theta)$ is regarded as a random variable in the Bayesian paradigm. First, prior distribution $\pi(\theta)$ is assumed to represent knowledge about $\theta$ prior to obtaining objective information. After gathering data, the prior distribution $\pi(\theta)$ is updated to the posterior distribution $\pi(\theta(x)$ by incorporating observations $x$ via the likelihood function $f(x \mid \theta)$ :

$$
\pi(\theta \mid x)=\frac{f(x \mid \theta) \pi(\theta)}{\int_{\Theta} f(x \mid \theta) \pi(\theta) d \theta}
$$

where $\theta$ denotes a set of model parameters introduced to fit the model to the data. The denominator on the right-hand side of Eq. (1) is the marginal distribution for the related random variable $X$, which is obtained by integrating out $\theta$ from the joint distribution of $X$ and $\theta$. The marginal distribution normalizes the product of the likelihood and prior distribution, the posterior distribution $f(\pi \mid \theta)$ is a valid probability density function (PDF). In this study, Markov chain Monte Carlo methodology (MCMC) is applied. The principle of MCMC is to create a target distribution by generating a sequence of points through a long run of simulations such as Markov chains. Markov chains can be constructed using MetropolisHastings algorithm.

\subsection{Traditional Fragility Function}

Traditional fragility modeling of bridges commonly used in prior studies [1-3] requires probabilistic seismic demand models (PSDMs) and limit state models. Component fragility curves are derived through the convolution of a PSDM and capacity-based limit state models $(C)$. The PSDM is a linear regression model in the log-transformed space for seismic demand $(D)$-IM pairs for each component (demand model conditioned on the IM). As a result, the fragility function for the $k$ th bridge component $\left(P F_{k \mid I M}\right)$ can be written:

$$
P F_{k \mid M}=P[D \geq C \mid I M]=\Phi\left[\frac{\ln \left(S_{D} / S_{C}\right)}{\sqrt{\beta_{D \mid M}^{2}+\beta_{C}^{2}}}\right]
$$

Where $S_{D}$ is the median of the seismic demand conditioned on the IM $\left(S_{D}=a I M^{b}\right)$, and $\beta_{D \mid I M}$ is the dispersion of the seismic demand. $\ln (a)$ and $b$ are the intercept and slope, respectively, of the linear regression of $\ln (D)$ on $\ln (I M) . S_{C}$ and $\beta_{C}$ are the median value and dispersion, respectively, of the capacity. $N$ is the number of simulations and $\Phi[\bullet]$ is the normal CDF.

\subsection{Fragility Function Employing Bayesian Parameter Estimation Method}

Previous studies $[4,5]$ recently derived parameterized fragility curves of bridges using multi-parameter demand models in conjunction with logistic regression techniques. A main characteristic of the fragility model is the utilization of the uncertainty parameters in demand models, in addition to an IM and logistic regression. The logistic regression model offers the form of CDF that describes bridge failure probabilities given the set of predictor parameters. The parameterized fragility model has several advantages over traditional fragility models conditioned only on the IM; (1) failure probability for a given IM can be easily estimated by substituting uncertainty (modeling) parameters when their specific value is available (called bridge-specific fragility), (2) the bridge-specific fragility model enables efficient and reliable predictions of fragility estimates with low computational cost, which may be incorporated into regional risk assessment packages, and (3) the sensitivity analysis can be performed to evaluate the effect of a modeling parameter on bridge fragilities, and (4) if the uncertainty parameters are probabilistic in nature (for fragility estimates of a bridge class), bridge-class fragility curves 
(conditioned only on the IM) can be developed by integrating the parameterized fragility function over the domain of the statistical distribution of the uncertainty parameters. This integration yields a form of the traditional fragility function, specifically failure probability as a function of only IM. The parameterized fragility function for the $k$ th component can be written as:

$$
P F_{k \mid I M, p_{1}, p_{2}, \cdots, p_{m}}=\frac{e^{b_{k, 0}+b_{k, I M} \cdot I M+\sum_{j=1}^{m} b_{k, j} \cdot p_{j}}}{1+e^{b_{k, 0}+b_{k, I M} \cdot I M+\sum_{j=1}^{m} b_{k, j} \cdot p_{j}}}
$$

Where $p_{j}(j=1, \ldots, m)$ is the input predictor variable, $b_{k, 0}, b_{k, I M}$, and $b_{k, j}$ 's $(j=1, \ldots, m)$ are the logistic regression coefficients for the $k$ th bridge component. If the values of all uncertainty parameters are known, Eq. (3) is considered the bridge-specific fragility function. Additionally, the bridge-class fragility function for the $k$ th component can defined as a function of $I M$.

$$
P F_{k \mid M}=\iint_{p_{1}} \cdots \int_{p_{m}} P F_{k \mid M, p_{1}, p_{2}, \cdots, p_{m}} f\left(p_{1}\right) f\left(p_{2}\right) \cdots f\left(p_{m}\right) d p_{1} d p_{2} \cdots d p_{m}
$$

Where $f\left(p_{1}\right), \ldots, f\left(p_{\mathrm{m}}\right)$ are the marginal PDFs for the uncertainty parameters. The detailed description can be found in [7].

\section{Case-Study Bridges}

\subsection{Description of As-Built Bridges}

To develop fragility curves of curved bridges for a regional seismic risk assessment (based on a bridge inventory analysis), this study selects the class of seven-span reinforced concrete box-girder bridges, one of typical configurations of curved concrete bridges in California. The bridge has single column bents, diaphragm abutments, and in-span hinges. Figure 2 shows the elevation of the case-study bridge, definition of bridge angle $\left(\alpha_{b}\right)$, and cross-sectional property of its members. In-span hinges are located at the one-fifths main-span length away from Pier 3 and Pier 5. In each in-span hinge, between the shear key and the deck, and between the adjacent decks lie a transverse and longitudinal gap, respectively.
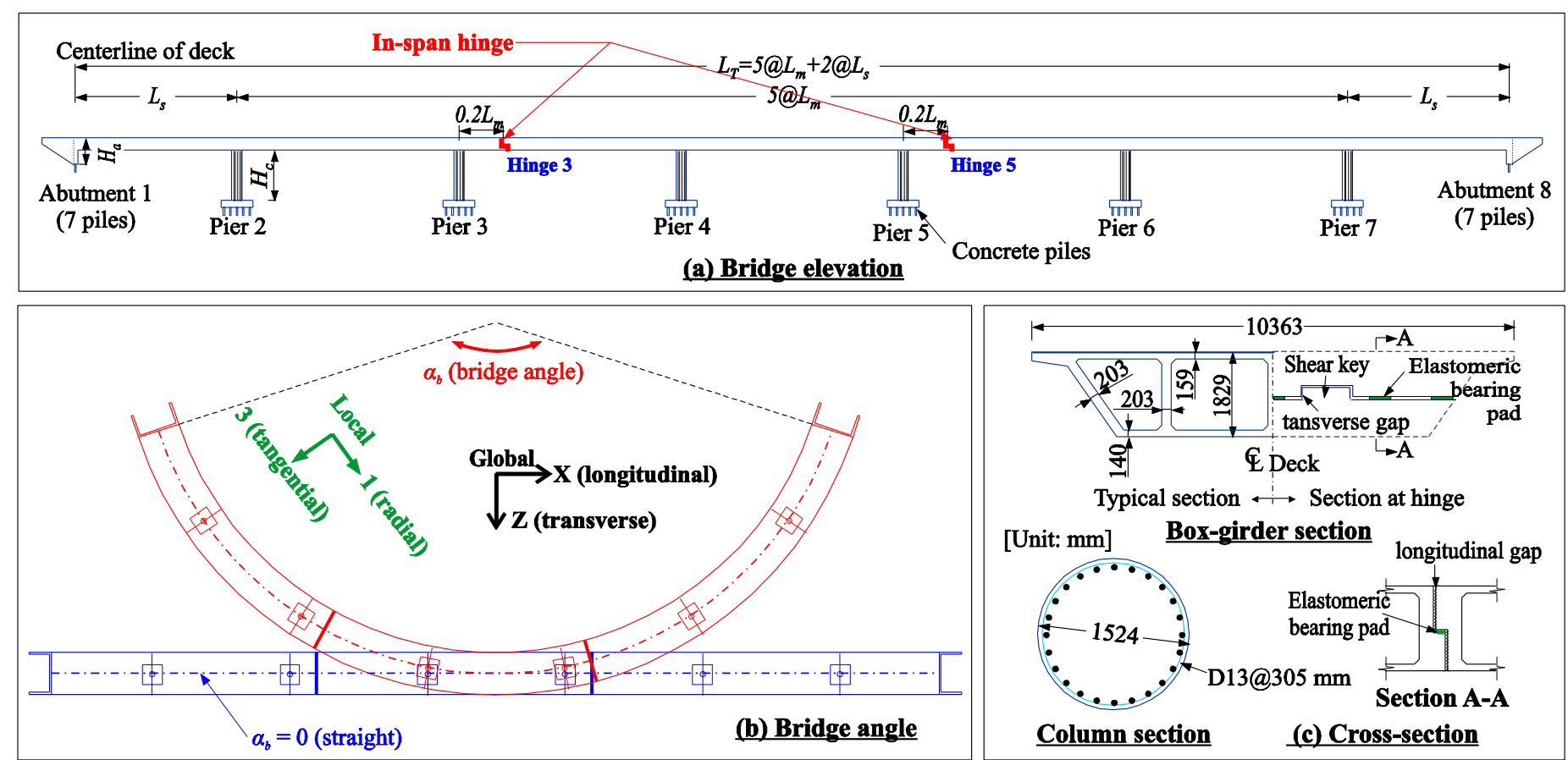

Fig. 1: Drawing of case-study bridge. 


\subsection{Geometric and Material Uncertainties}

To facilitate a regional seismic risk assessment, geometric and material uncertainties are accounted for in this study and are summarized in Table 1. Most of the parameters are determined based on the plan review of more than 1,000 bridges using the in-house database obtained from the Caltrans [8]. The bridge angle $\left(\alpha_{b}\right)$ was not well-defined from the plan review whereas other parameters were relatively well-identified. The bridge angle is defined as the angle between the start and end points of the superstructure. Following the work of [9], this study treats the bridge angle as a random variable with a uniform distribution $[0,3]$. The maximum value is restricted to $3.0 \mathrm{rad}$ to reflect realistic conditions of the bridges. A set of three-dimensional bridge models is created by sampling across the range of modeling parameters (Table 1) via a Latin Hypercube Sampling (LHS) technique, and are then paired randomly with ground motions.

Table 1: Uncertainty (modeling) parameters and their probability distribution.

\begin{tabular}{|c|c|c|c|c|c|}
\hline \multirow{3}{*}{ Parameter } & \multicolumn{5}{|c|}{ Probability distribution } \\
\hline & \multirow[t]{2}{*}{ Type $^{\S}$} & \multicolumn{2}{|c|}{ Parameters $^{\dagger}$} & \multicolumn{2}{|c|}{ Truncated limit } \\
\hline & & $\alpha$ & $\beta$ & Lower & Upper \\
\hline \multicolumn{6}{|l|}{ Material properties } \\
\hline Concrete compressive strength, $f_{c}(\mathrm{MPa})$ & $\mathrm{N}$ & 26.9 & 3.3 & 20.3 & 35.8 \\
\hline Rebar yield strength, $f_{y}(\mathrm{MPa})$ & $\mathrm{LN}$ & 5.98 & 0.08 & 338 & 464 \\
\hline Shear modulus of elastomeric bearings, $G_{p}(\mathrm{MPa})$ & $\mathrm{U}$ & 0.55 & 1.72 & - & - \\
\hline Coefficient of friction of elastomeric bearings, $\mu_{p}$ & $\mathrm{~N}$ & 0.3 & 0.1 & 0.1 & 0.5 \\
\hline \multicolumn{6}{|l|}{ Superstructure } \\
\hline Main-span length, $L_{m}(\mathrm{~mm})$ & $\mathrm{LN}$ & 10.17 & 0.25 & 15850 & 43282 \\
\hline Ratio of side-span to main-span length, $\left(\eta=L_{s} / L_{m}\right)$ & $\mathrm{N}$ & 0.57 & 0.17 & 0.22 & 0.92 \\
\hline Bridge angle, $\alpha_{b}(\mathrm{rad})$ & $\mathrm{U}$ & 0 & 3.0 & - & - \\
\hline \multicolumn{6}{|l|}{ Interior bent (single-column) } \\
\hline Column clear height, $H_{c}(\mathrm{~mm})$ & $\mathrm{LN}$ & 8.79 & 0.13 & 5029 & 8534 \\
\hline Column longitudinal reinforcement ratio, $\rho_{c}$ & $\mathrm{U}$ & 0.01 & 0.04 & - & - \\
\hline Translational stiffness of a pile group, $K_{f t}\left(\times 10^{5} \mathrm{~N} / \mathrm{mm}\right)$ & $\mathrm{N}$ & 2.45 & 1.05 & 1.05 & - \\
\hline Rotational stiffness of a pile group, $K_{f r}\left(\times 10^{12} \mathrm{~N}-\mathrm{mm} / \mathrm{rad}\right)$ & $\mathrm{N}$ & 7.34 & 1.13 & 5.65 & - \\
\hline \multicolumn{6}{|l|}{ Exterior bent (diaphragm abutment on piles) } \\
\hline Abutment height, $H_{a}(\mathrm{~mm})$ & $\mathrm{LN}$ & 8.07 & 0.15 & 2438 & 4267 \\
\hline Backfill type, $B T$ (sand vs. clay) & B & - & - & - & - \\
\hline Pile stiffness, $k_{p}(\mathrm{~N} / \mathrm{mm})$ & $\mathrm{LN}$ & 9.55 & 0.08 & - & - \\
\hline \multicolumn{6}{|l|}{ Gap } \\
\hline Longitudinal (pounding), $\Delta_{l}(\mathrm{~mm})$ & $\mathrm{LN}$ & 3.03 & 0.5 & 7.6 & 55.9 \\
\hline Transverse (shear key), $\Delta_{t}(\mathrm{~mm})$ & $\mathrm{LN}$ & 2.53 & 0.2 & 8.4 & 19 \\
\hline \multicolumn{6}{|l|}{ Other parameters } \\
\hline Mass factor, $m_{f}$ & $\mathrm{U}$ & 1.1 & 1.4 & - & - \\
\hline Damping, $\xi$ & $\mathrm{N}$ & 0.045 & 0.0125 & 0.02 & 0.07 \\
\hline Earthquake direction (fault normal FN vs. parallel FP), ED & B & - & & - & - \\
\hline
\end{tabular}

$\S \mathrm{N}=$ normal, $\mathrm{LN}=$ lognormal, $\mathrm{U}=$ uniform, and $\mathrm{B}=$ Bernoulli distribution.

$\dagger \alpha$ and $\beta$ represent parameters of the respective distribution. These denote mean and standard deviation for a normal distribution, lower and upper bound in the case of uniform distribution and mean and standard deviation of the associated normal distribution in the case of a lognormal distribution.

\subsection{Modeling Assumptions}

Following the recommendations of [10], this study models the deck in one span as a spine with several elastic beamcolumn elements along the bridge centerline: eight and ten elements per side (outer) and main (interior) span, respectively (Fig. 3). This figure illustrates the numerical model of multiple components in the curved bridges built in OpenSees [6]. Per [11], the effective flexural stiffness for decks is assumed to be $62.5 \%$ of gross stiffness to account for cracking. To represent the diaphragms and in-span hinges and to capture the torsion of the box-girder due to the bridge curvature, the 
transverse beam elements are modeled using elastic beam-column elements (rigid and massless). The vertical translational masses are assigned to the ends of the outriggers in order to represent the rotational mass moment of inertia of the deck [3]. Columns are modeled using nine fiber-type displacement-based beam-column elements along with rigid links at the deckcolumn and footing-column connections. In the fiber sections, the OpenSees Hysteretic and Concrete02 material models are used to simulate the longitudinal reinforcement and unconfined and confined concrete. The confined concrete is simulated using the model of [12]. Each pile foundation is modeled using four lumped linear springs: two translational springs, and two rotational springs. Each in-span hinge has five bearing elements (radial and tangential), five pounding elements (radial), and one shear key element (tangential). The nonlinear response of elastomeric bearings is simulated employing an elastic-perfectly-plastic model. Additionally, the nonlinear response of an interior shear key is simulated based on experimental results by [13]. The pounding effect is simulated using a nonlinear compression element with the gap being modeled as proposed by [14]. Each abutment is simulated using five zero-length nonlinear elements to capture the inelastic response for the radial and tangential directions. It is assumed that the passive and active resistances (radial direction) are provided by the composite action of soil and piles, and piles alone, respectively. The tangential resistance is assumed to be provided by the piles. The backfill soil and pile responses are simulated using the hyperbolic soil model of [15] and the trilinear spring model, respectively. The detailed description of modeling techniques can be found in [3].

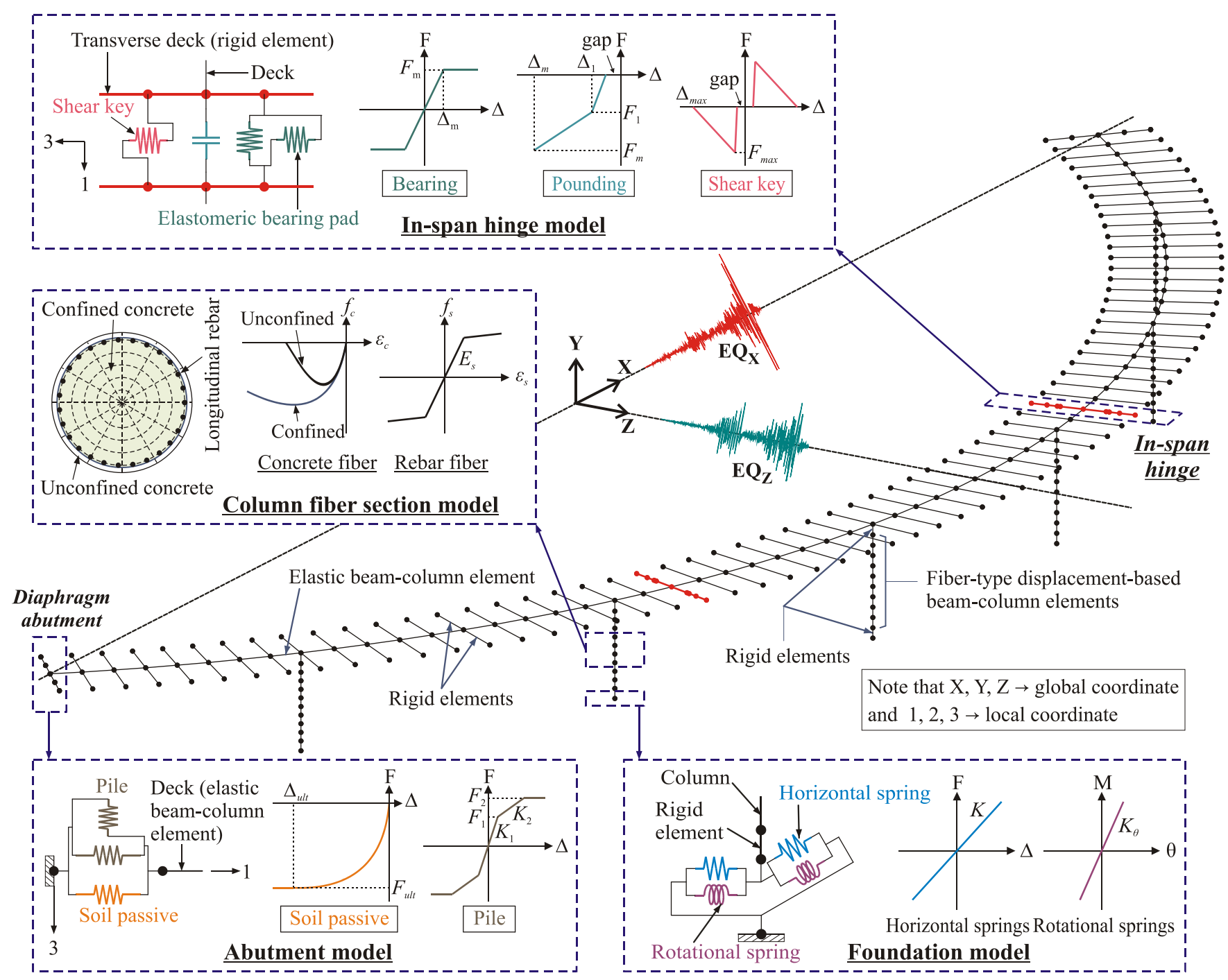

Fig. 2: Numerical model of case-study bridge [3]. 


\subsection{Ground Motion Suite}

The ground motion suite must include a wide range of IMs representative of seismic hazard at the area of interest. To achieve this aim, this study selects the suite of ground motions developed by Baker et al. [16], which was proposed as part of the PEER Transportation Research Program. The suite comprises 120 pairs of broad-band ground motions and 40 pairs of near fault ground motions. To better understand the response of bridge components under high intensity ground motion, the entire suite of 160 motions is scaled by a factor of 1.5 and 2.0. An expanded suite of 480 ground motions is used for the fragility assessment in this study. Per Ramanathan et al. [2], this incidence angle of seismic loads is assumed to follow a Bernoulli distribution (see Table 1).

\subsection{Engineering Demand Parameters and Associated Limit State Models}

To estimate the vulnerability of the case-study bridge, four bridge components are considered in this study; column, superstructure, bearing, and abutment. For these components, six engineering demand parameters (EDPs) associated with their seismic demands monitored in dynamic analyses are selected; peak column curvature ductility $\left(\mu_{\phi}\right)$, peak unseating deformation $\left(\delta_{u}\right.$ in $\left.\mathrm{mm}\right)$, and peak bearing shear strain $\left(\gamma_{b}\right.$ in $\left.\%\right)$ as well as peak radial (passive and active), and tangential abutment deformations $\left(\delta_{p}, \delta_{a}\right.$, and $\delta_{t}$ in $\left.\mathrm{mm}\right)$. For each EDP, limit state models follow a two-parameter lognormal distribution (median $S_{C}$ and dispersion $\beta_{C}$ ) and are summarized in Table 2.

Table 2: Limit state models for EDPs of bridge components [2].

\begin{tabular}{|c|c|c|c|c|c|c|c|c|c|}
\hline \multirow{2}{*}{\multicolumn{2}{|c|}{ Component }} & \multicolumn{2}{|c|}{$\mathrm{LS}_{1}$} & \multicolumn{2}{|c|}{$\mathrm{LS}_{2}$} & \multicolumn{2}{|c|}{$\mathrm{LS}_{3}$} & \multicolumn{2}{|c|}{$\mathrm{LS}_{4}$} \\
\hline & & $S_{C}$ & $\beta_{C}$ & $S_{C}$ & $\beta_{C}$ & $S_{C}$ & $\beta_{C}$ & $S_{C}$ & $\beta_{C}$ \\
\hline \multicolumn{2}{|c|}{ Colum curvature ductility, $\mu_{\phi}$} & 1.0 & 0.35 & 2.0 & 0.35 & 3.5 & 0.35 & 5.0 & 0.35 \\
\hline \multicolumn{2}{|c|}{ Unseating displacement, $\delta_{u}(\mathrm{~mm})$} & - & - & - & - & 152 & 0.35 & 305 & 0.35 \\
\hline \multicolumn{2}{|c|}{ Bearing shear strain, $\gamma_{b}(\%)$} & 100 & 0.35 & 300 & 0.35 & - & - & - & - \\
\hline \multirow{3}{*}{$\begin{array}{l}\text { Abutment } \\
\text { displacement (mm) }\end{array}$} & Passive action, $\delta_{p}$ & 76 & 0.35 & 254 & 0.35 & - & - & - & - \\
\hline & Active action, $\delta_{a}$ & 38 & 0.35 & 102 & 0.35 & - & - & - & - \\
\hline & Tangential action, $\delta_{t}$ & 25 & 0.35 & 102 & 0.35 & - & - & - & - \\
\hline
\end{tabular}

\section{Application of Bayesian Parameter Estimation to Bridge Fragilities}

\subsection{Multi-Parameter Demand Models}

Each demand model is developed using input variables and an output variable obtained from dynamic analyses. To develop the multi-parameter demand model of bridge components, 480 bridge realizations using uncertainty values sampled from the LHS technique are built using OpenSees [6]. Each bridge sample is randomly paired with one of the ground motions. For each bridge-ground motion pair, a dynamic analysis is performed to monitor peak responses of bridge components, which are adopted as EDPs in this study. Also, the PGA is adopted as IM. In this study, the geometric mean of two horizontal PGAs is used as the PGA. Using 480 pairs of input predictor variables (a total of 20) and response data for six EDPs, demand models are constructed using an MCMC-based Bayesian parameter estimation method. By taking logarithms for the input parameters and response data, the multi-parameter demand model can be expressed as:

$$
\ln (y)=\beta_{0}+\beta_{P G A} \cdot \ln (P G A)+\sum_{i=1}^{m} \beta_{i} \cdot \ln \left(p_{i}\right)+\sigma \varepsilon
$$

where $m=$ number of uncertainty parameters presented in Table 1 (here, $m=19), p_{1}=E D, p_{2}=f_{c}(\mathrm{MPa}), p_{3}=f_{y}(\mathrm{MPa}), p_{4}$ $=\xi, p_{5}=m_{f}, p_{6}=\mu_{p}, p_{7}=G_{p}(\mathrm{MPa}), p_{8}=\Delta_{l}(\mathrm{~mm}), p_{9}=\Delta_{t}(\mathrm{~mm}), p_{10}=H_{a}(\mathrm{~mm}), p_{11}=B T, p_{12}=k_{p}(\mathrm{~N} / \mathrm{mm}), p_{13}=\rho_{c}, p_{14}=$ $K_{f r}(\mathrm{~N}-\mathrm{mm} / \mathrm{rad}), p_{15}=K_{f t}(\mathrm{~N} / \mathrm{mm}), p_{16}=\alpha_{b}(\mathrm{rad}), p_{17}=L_{m}(\mathrm{~mm}), p_{18}=\eta$, and $p_{19}=H_{c}(\mathrm{~mm})$. The abutment soil backfill $(B T)$ and earthquake direction $(E D)$ follow the Bernoulli distribution. For convenience, $B T=e$ (where $e$ is the Euler's number) if the backfill is sand. Otherwise, $B T=e^{2}\left(\ln (e)=1\right.$ and $\left.\ln \left(e^{2}\right)=2\right)$. In the same fashion, if the fault normal 
component of an earthquake is applied to the global X-axis (longitudinal) shown in Figs. 1 and $2, E D=e$. Otherwise, $E D=$ $e^{2}$. Additionally, $\theta_{i}$ 's $\left(\beta_{i}\right.$ and $\left.\sigma\right)$ are defined by the posterior mean of the regression coefficients.

Using the MCMC-based Bayesian approach, the posterior mean and COV of $\theta_{i}$ 's for all bridge components are computed. The posterior means of $\beta_{i}$ 's are used to construct the demand model of each EDP. Note that if the regression coefficient for a predictor variable in the demand models is positive, the seismic demand increases, resulting in the increase in the failure probability.

\subsection{Parameterized Fragility Curves via Logistic Regression}

Based on the multi-parameter demand model and the limit state models, a large number of demands and capacities $\left(N_{\text {logistic }}=2 \times 10^{5}\right.$ in this study) are generated based on the statistical distribution of input parameters (uncertainty parameters presented in Table 1 and PGA) via an LHS-based experimental design. Pairs of the sampled demand and capacity estimate for each bridge component at a specific limit state are compared to obtain a binary survival-failure vector, which is used in a logistic regression analysis. The resulting regression coefficients are used to construct the parameterized fragility curve for the component at the limit state. A parameterized fragility function for a certain limit state of the $k$ th component $\left(P F_{k \mid I M, p_{1}, p_{2}, \cdots, p_{m}}\right)$ can be expressed in a form of logistic regression:

$$
\ln \left(\frac{P F_{k \mid P G A, p_{1}, p_{2}, \cdots, p_{m}}}{1-P F_{k \mid P G A, p_{1}, p_{2}, \cdots, p_{m}}}\right)=b_{k, 0}+b_{k, P G A} \cdot \ln (P G A)+\sum_{i=1}^{m} b_{k, i} \cdot \ln \left(p_{i}\right)
$$

All variables in Eq. (7) are already defined in Section 2. If all the input parameters are specified and then substituted into the model, the bridge-specific fragility curves can be derived. Additionally, the effect of uncertainty parameters on bridge vulnerability can be investigated by varying one parameter each time. Moreover, the bridge-specific fragility function can be used to develop fragility curves for multi-frame concrete box-girder bridges with different material properties and configurations located at the California region if the uncertainty parameters are available.

As mentioned above, bridge-class fragility curves can be derived by integrating parameterized fragility functions over the domain of the statistical distribution of uncertainty parameters, as presented in Eq. (4). To compute higher dimensional integrals, this study adopts a Monte Carlo integration that numerically calculates a definite integral using random numbers. The random numbers are sampled using the statistical distribution of uncertainty parameters with lower and upper bounds presented in Table 1 via the LHS technique. For each parameter, the number of samples is determined as 20,000 for each increment of PGA. The integrated results yields a bridge-class fragility curve conditioned only on PGA. This bridge-class fragility curve can be used when the vulnerability of a bridge class (not a specific bridge) is assessed. In this case, the proposed fragility curve is used to compare a traditional fragility curve using Eq. (2). Figure 3 compares the fragility curves for the columns and abutment active actions using the proposed and traditional fragility models. The figures indicate that the curve slope for the proposed fragility model is steeper, indicating that the failure probabilities decrease at lower PGAs and increase at higher PGAs. Thus, the proposed model can significantly reduce the model error by accounting for uncertainty parameters in addition to the IM.

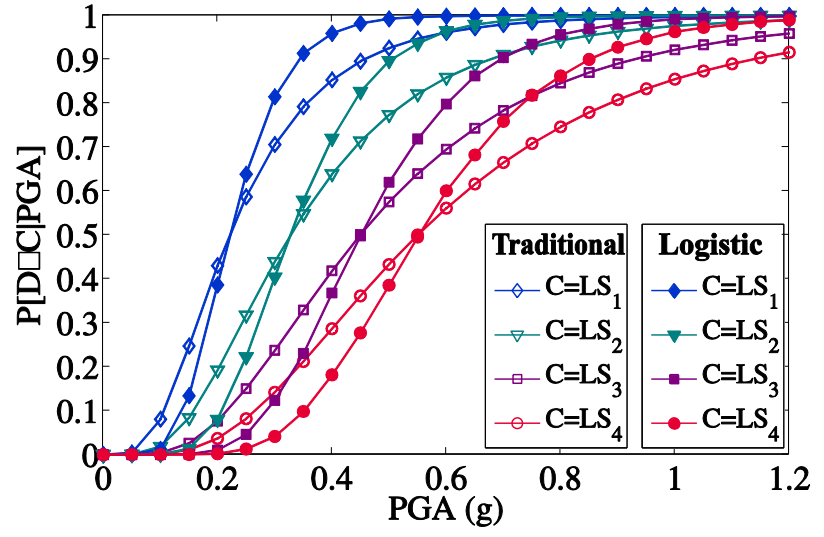

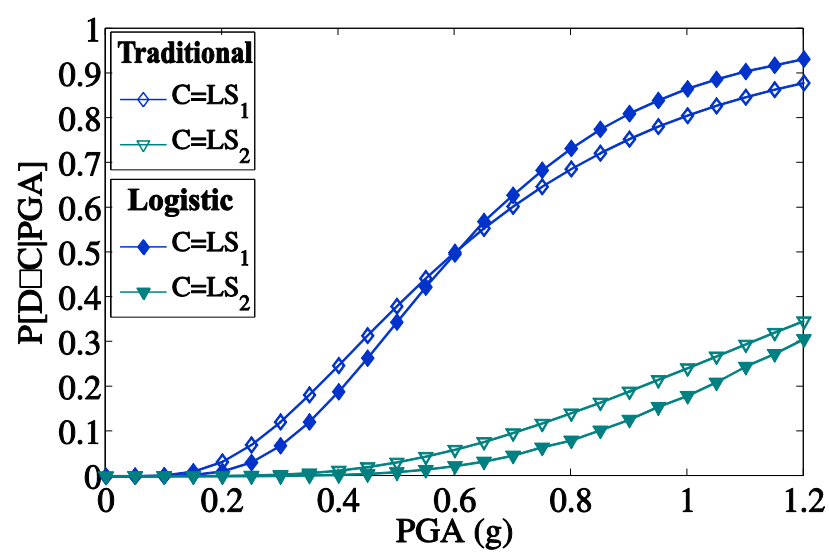




\section{Conclusion}

Fig. 3: Comparison of bridge-class column fragility curves.

This study proposes a probabilistic framework for facilitating the seismic risk assessment of bridges with the same bridge class in the transportation network at the specific area. This framework includes the selection of a bridge class, characterization of bridge attributes such as material and geometric uncertainties, creation of numerical component models, construction of multi-parameter demand models using a Bayesian parameter estimation method, and development of bridge-class fragility models using logistic regression. The parameterized fragility models are used (1) to produce bridgespecific (one-dimensional) fragility curves when the uncertainty parameters are available and (2) to develop bridge-class (one-dimensional) fragility curves using a Monte Carlo integration. Additionally, the Bayesian approach facilitates identifying significant uncertainty parameters that affect seismic demands without performing numerous structural analyses required for design of experiments. Comparison of the proposed and traditional fragility models indicates that the traditional fragility model overestimates failure probabilities at lower PGAs and underestimates them at higher PGAs. This proposed framework can be readily implemented into other bridge classes if statistical distributions of uncertainty parameters based on a bridge inventory analysis are available.

\section{Acknowledgements}

This research was supported by Basic Research Program in Science and Engineering through the National Research Foundation of Korea funded by the Ministry of Education (NRF-2016R1D1A1B03933842).

\section{References}

[1] B. G. Nielson and R. DesRoches, "Seismic fragility methodology for highway bridges using a component level approach,” Earthquake Eng. Struct. Dyn., vol. 36, pp. 823-839, 2007.

[2] K. N. Ramanathan, J. E. Padgett, and R. DesRoches, "Temporal evolution of seismic fragility curves for concrete box-girder bridges in California," Eng. Struct., vol. 97, pp. 29-46, 2015.

[3] J. -S. Jeon, R. DesRoches, T. Kim, and E. Choi, "Geometric parameters affecting seismic fragilities of curved multiframe concrete box-girder bridges with integral abutments," Eng. Struct., vol. 122, pp. 121-143, 2016.

[4] J. Ghosh, J. E. Padgett, and L. Dueñas-Osorio, "Surrogate modeling and failure surface visualization for efficient seismic vulnerability assessment of highway bridges," Probabilist. Eng. Mech., vol. 34, pp. 189-199, 2013.

[5] K. Rokneddin, J. Ghosh, L. Dueñas-Osorio, and J. E. Padgett, "Seismic reliability assessment of aging highway bridge networks with field instrumentation data and correlated failures, II: Application," Earthquake Spectra, vol. 30, pp. 819-843, 2014.

[6] F. McKenna, "OpenSees: A framework for earthquake engineering simulation," Comput. Sci. Eng., vol. 13, pp. 5866, 2011.

[7] J. -S. Jeon, S. Mangalathu, J. Song, and R. DesRoches, "Parameterized seismic fragility curves for curved multiframe concrete bridges using Bayesian parameter estimation," J. Earthquake Eng., in review, 2016.

[8] Caltrans, Personal communication with the P266, Task 1780 Fragility project panel members including C Roblee, M Yashinsky, M Mahan, T Shantz, and L Turner, California Department of Transportation. Sacramento, CA, 2015.

[9] O. C. Celik and B. R. Ellingwood, "Seismic fragilities for non-ductile reinforced concrete frames-Role of aleatoric and epistemic uncertainties," Struct. Saf., vol. 32, pp. 1-12, 2010.

[10] Caltrans. (2016, October 5). Bridge design practice manual [Online]. Available: http://www.dot.ca.gov/hq/esc/techpubs/manual/bridgemanuals/bridge-design-practice/bdp.html

[11] Caltrans, Seismic design criteria version 1.7, Office of Structures Design, California Department of Transportation, Sacramento, CA, 2013.

[12] J. B. Mander, M. J. N. Priestley, and R. Park, "Theoretical stress-strain model for confined concrete," J. Struct. Eng., vol. 114, pp. 1804-1826, 1988.

[13] P. F. Silva, S. Megally, F. Seible, "Seismic performance of sacrificial interior shear keys," ACI Struct. J., vol. 100, 177-187, 2003.

[14] S. Muthukumar and R. DesRoches, "A Hertz contact model with non-linear damping for pounding simulation," Earthquake Eng. Struct. Dyn., vol. 35, pp. 811-828, 2006. 
[15] A. Shamsabadi, P. Khalili-Tehrani, J. P. Stewart, E. Taciroglu, "Validated simulation models for lateral response of bridge abutments with typical backfills," J. Bridge Eng., vol. 15, pp. 302-311, 2010.

[16] J. W. Baker, S. K. Shahi, and N. Jayaram, New ground motion selection procedures and selected motions for the PEER Transportation Research Program, Pacific Earthquake Engineering Research Center, University of California, Berkeley, CA, PEER Report 2011/03, 2011. 